\title{
Effect of heme oxygenase 1 and renin/prorenin receptor on oxidized low-density lipoprotein-induced human umbilical vein endothelial cells
}

\author{
XIN GONG $^{1 *}$, CONGYANG LIU ${ }^{1 *}$, HONGLING WANG ${ }^{2}$, JINYING FAN $^{3}$, \\ CUIHONG JIANG ${ }^{1}$ and YONG ZOU ${ }^{1}$ \\ ${ }^{1}$ Department of Integrated Chinese and Western Medicine; ${ }^{2}$ Central Laboratory, The Affiliated Yantai \\ Yuhuangding Hospital of Qingdao University; ${ }^{3}$ Department of Cardiology, \\ Yantaishan Hospital, Yantai, Shandong 264000, P.R. China
}

Received August 3, 2018; Accepted April 12, 2019

DOI: $10.3892 /$ etm.2019.7769

\begin{abstract}
The incidence of depression has previously been correlated to hypertension. The aim of the present study was to explore the mechanisms of depression and hypertension by examining the expression and interaction of renin/prorenin receptor (PRR) and heme oxygenase $1(\mathrm{HO}-1)$ in vascular endothelial cells. A case-control study was conducted, and general data and serum factors were compared between hypertension patients complicated with depression and patients with hypertension alone. Logistic regression analysis was used to detect risk factors associated with hypertension complicated with depression. In addition, human umbilical vein endothelial cells (HUVECs) were treated with oxidized low-density lipoprotein (ox-LDL) and/or PRR gene silencing, and a Cell Counting Kit-8 (CCK-8) assay was performed to test their proliferation. The concentrations of inflammatory factors and oxidative stress factor were also detected using enzyme-linked immunosorbent assay and chemical colorimetry. Western blot analysis and reverse transcription-quantitative polymerase chain reaction were applied to detect protein and mRNA expression levels, respectively. The results revealed that HO-1 and renin precursor (Rep) were independent factors that affected hypertension complicated with depression. Serum HO-1 levels in patients with hypertension complicated with depression were significantly lower than that in hypertensive patients without depression, while Rep levels in patients with hypertension complicated with
\end{abstract}

Correspondence to: Dr Yong Zou, Department of Integrated Chinese and Western Medicine, The Affiliated Yantai Yuhuangding Hospital of Qingdao University, 20 East Yuhuangding Road, Yantai, Shandong 264000, P.R. China

E-mail: yongzou_zy@163.com

*Contributed equally

Key words: hypertension, depression, renin/prorenin receptor, human umbilical vein endothelial cells depression were significantly higher than that in hypertensive patients without depression. In HUVECs, ox-LDL reduced the cell proliferation in a dose-dependent manner, upregulated the expression of PRR gene and downregulated the expression of HO-1 gene. It was also observed that silencing of the PRR gene promoted the expression of the HO-1 gene. Furthermore, ox-LDL upregulated the inflammatory response and oxidative stress levels, while PRR gene silencing inhibited the ox-LDL-induced inflammatory factor and oxidative stress levels in HUVECs. Thus, regulating the expression levels of HO-1 and PRR to inhibit the oxidative stress and pro-inflammatory effect of ox-LDL may provide new insight for the treatment of hypertension patients with depression.

\section{Introduction}

Hypertension is a common condition associated with the occurrence of cardiovascular and numerous other diseases. The association between hypertension and depression has been studied for over a century $(1,2)$. Although the results of such studies have been controversial and the underlying mechanisms involved are not well defined, the great number of clinical, epidemiological and biological evidence available is sufficient to confirm the interaction between depression and hypertension $(3,4)$.

Vascular endothelial cells (VECs) are in direct contact with blood flow and serve as a permeability barrier between vascular wall tissue and blood $(5,6)$. Abnormalities in the function of VECs are associated with the development of various cardiovascular diseases. In addition, oxidative stress and stimulation of inflammatory factors can cause endothelial dysfunction (7). Studies have confirmed that oxidative stress and inflammatory factors in hypertension and depression patients were increased when compared with those in healthy individuals (8-11).

The renin-angiotensin system (RAS) is involved in the regulation and maintenance of the balance of blood pressure, water and electrolytes. It is also one of the most important systems that regulate VECs (12). Under pathological conditions, RAS is involved in the pathogenesis of hypertension (13). In recent 
years, it has been reported that RAS is also associated with the occurrence and development of depression (14).

Renin/prorenin receptor (PRR) is a co-receptor of renin and renin precursor (Rep). When combined with its ligand, PRR promotes the production of angiotensin II (Ang II), which leads to vasoconstriction and elevated blood pressure, as well as to inflammation, causing damage to VECs $(15,16)$. Oxidized low-density lipoprotein (ox-LDL), which is an important factor in the damage of VECs, is produced in large quantities under high-oxidative stress level, and can cause inflammatory reaction and destroy the vascular endothelium (17). Heme oxygenase 1 (HO-1) is the rate-limiting enzyme in the process of heme catabolism and the most important player against oxidative stress in mammals $(17,18)$. However, to the best of our knowledge, no research has been conducted to date on HO-1 and PRR in hypertension complicated with depression.

Although a significant correlation between depression and hypertension has been reported in clinical phenotypes, there is a lack of clear evidence on the underlying mechanism. In the present study, a case-control study was first conducted in patients with hypertension alone or hypertension complicated with depression, in order to explore the factors affecting hypertension with depression. Subsequently, the expression and interaction of associated independent risk factors were investigated in human umbilical vein endothelial cells (HUVECs). The study focused on the mechanism of depression and hypertension comorbidity by studying the expression and interaction of PRR and HO-1 in HUVECs.

\section{Materials and methods}

Patients. A case-control study was conducted to investigate the influencing factors of hypertension complicated with depression. Between February 2013 and October 2016, 85 patients (including 45 males and 40 females, with a mean age of $53.13 \pm 5.24$ years) with both hypertension and depression were selected and classified as the observation group. Another 85 patients (including 44 males and 41 females, with a mean age of $53.82 \pm 5.33$ years) with hypertension without depression were assigned to the control group. The patients were diagnosed with hypertension using the ICD-10 categories I10.x, and diagnosed with depression using the ICD-10 categories F32.x and F33.x (19). The hypertension patients complicated with depression met the aforementioned diagnostic criteria for the two diseases. However, patients with comorbidity of various infections, diabetes, malignant tumors, stroke, immune system diseases and nervous system diseases, or patients taking angiotensin-converting enzyme inhibitors and angiotensin receptor antagonists to reduce blood pressure were excluded from the present study. The study was approved by the Ethics Committee of the Affiliated Yantai Yuhuangding Hospital of Qingdao University (Yantai, China), and informed consent was signed by each participant. Patient information, such as age, sex and serum-associated factors, was collected from their medical records and compared. Logistic regression analysis was used to detect risk factors for hypertension patients complicated with depression. Serum was obtained from fasting blood samples via centrifugation at 2,500 x g for $10 \mathrm{~min}$ at $4^{\circ} \mathrm{C}$. Samples were aliquoted and stored at $-80^{\circ} \mathrm{C}$ until required. Blood pressure was measured via a mercury column desktop sphygmomanometer. HO-1 and Rep from serum were assayed using a human HO-1 ELISA kit (cat. no. SEA584Hu; Cloud-Clone Corp., Houston, USA) and a human renin precursor ELISA kit (cat. no. CSB-E13181 h; Cusabio Technology LLC). Levels of cortisol, triglyceride, total cholesterol, low-density lipoprotein cholesterol, high density lipoprotein cholesterol, creatinine, alanine aminotransfease and glutamic oxalacetic transaminase were detected using an Automated hematology analyzer (PUZS-300; Perlong Medical Equipment Co., Ltd.).

Cell culture and observation. Newborn human umbilical cord specimens of newborns were obtained from the Affiliated Yantai Yuhuangding Hospital of Qingdao University (Yantai, China). The present study was approved by the Ethics Committee of the Affiliated Yantai Yuhuangding Hospital of Qingdao University and informed consent was obtained from all patients. HUVECs were prepared from umbilical cord veins by collagenase digestion, as described by Jaffe et al (20). HUVECs were then cultured in RPMI-1640 medium containing $10 \%$ fetal bovine serum with $100 \mathrm{U} / \mathrm{ml}$ penicillin and $100 \mu \mathrm{g} / \mathrm{ml}$ streptomycin (all, Gibco; Thermo Fisher Scientific, Inc.) in an incubator with $5 \% \mathrm{CO}_{2}$ at $37^{\circ} \mathrm{C}$.

According to different experimental groups, culture was conducted in the presence of corresponding concentrations of ox-LDL (Sigma-Aldrich; Merck KGaA). To explore the effects of ox-LDL on HUVECs, in addition to the control group (without ox-LDL treatment), cells were divided into four groups according to the concentration of ox-LDL administered $(10,50,100$ and $150 \mu \mathrm{g} / \mathrm{ml}$ groups). Following treatment, cell proliferation was detected at $0,24,48$ and $72 \mathrm{~h}$ in each group using a cell counting kit-8 (CCK-8) assay as described below.

Immunocytochemistry. The expression of factor VIII in HUVECs was detected using an immunofluorescence technique. HUVECs were fixed with $4 \%$ paraformaldehyde at room temperature for $20 \mathrm{~min}$ on glass and washed three times with PBS (each, 3 min). PBS containing 0.2\% Triton X-100 (Thermo Fisher Scientific, Inc.) was subsequently used for the permeabilization of cells for $15 \mathrm{~min}$. Cells were blocked with $5 \%$ bovine serum albumin (Thermo Fisher Scientific, Inc.) at room temperature for $15 \mathrm{~min}$ and incubated with anti-factor VIII antibodies (cat. no. ab78852; 1:100; Abcam) at $4^{\circ} \mathrm{C}$ overnight. After washing with PBS, HUVECs were incubated with FITC-conjugated goat anti-mouse IgG H\&L (cat. no. ab6785; 1:5,000; Abcam) for $1 \mathrm{~h}$ at $37^{\circ} \mathrm{C}$ and then further incubated with DAPI (Thermo Fisher Scientific, Inc.) at room temperature for $10 \mathrm{~min}$. The results were observed under a MF22 fluorescence microscope (magnification, x200; Guangzhou Micro-shot Technology Co., Ltd.).

Transfection. For PRR silencing, $50 \mathrm{nM}$ small interfering (si)RNA targeting PRR (siPRR) was purchased from GenePharma Co., Ltd. And transfected using Lipofectamine ${ }^{\circledR}$ 2000 (Invitrogen; Thermo Fisher Scientific, Inc.). A mock group transfected with $2 \mu \mathrm{g} / \mathrm{ml}$ Lipo reagent (Invitrogen; Thermo Fisher Scientific, Inc.) and an untransfected control group were also established. The sequences of siPRR were as follows: 5'-CCUUGCGUAUAAGUAUAAUTT-3' (forward) and 5'-AUUAUACUUAUACGCAAGGTT-3' (reverse). 
Transfected cells were then incubated for $48 \mathrm{~h}$ at $37^{\circ} \mathrm{C}$. Following transfection for $48 \mathrm{~h}$, cells were harvested and the transfection efficiency was detected using reverse transcription-quantitative (RT-q) PCR and western blot analyses.

CCK-8 assay. A CCK-8 assay was conducted to test the cell proliferation of the different experimental groups at 0,24 , 48 and $72 \mathrm{~h}$, and the kit used was purchased from Dojindo Molecular Technologies, Inc. Briefly, cells $\left(2 \times 10^{3}\right.$ cells/well $)$ were seeded into 96-well plates and pre-incubated for $24 \mathrm{~h}$ at $37^{\circ} \mathrm{C}$ in an incubator with $5 \% \mathrm{CO}_{2}$. Subsequently, $10 \mu 1 \mathrm{CCK}-8$ reagent was added into each well and cultured for $4 \mathrm{~h}$ at $37^{\circ} \mathrm{C}$ in $5 \% \mathrm{CO}_{2}$. A microplate reader (ELX 800; BioTek Instruments, Inc.) was used to measure the optical density (OD) value of each well at $450 \mathrm{~nm}$.

Flow cytometry. Flow cytometry was used to detect the reactive oxygen species (ROS) levels using ROS assay kits purchased from BD Biosciences (BD Pharmingen). A total of $1 \times 10^{6}$ cells were washed with PBS at $4^{\circ} \mathrm{C}$ and resuspended to a concentration of $4 \times 10^{5}$ cells $/ \mathrm{ml}$. Subsequently, $10 \mu \mathrm{mol} / 1$ of the fluorescent probe DCFH-DA was added and incubated for 20 min. A flow cytometer (BD Biosciences) was the applied to analyze the ROS levels at an excitation wavelength of $488 \mathrm{~nm}$ and an emission wavelength of $525 \mathrm{~nm}$.

Enzyme-linked immunosorbent assay (ELISA) and chemical colorimetry. The concentration of interleukin (IL)- 6 (cat. no. CSB-E04638 h), IL-10 (cat. no. CSB-E04593 h), transforming growth factor (TGF)- $\alpha$ (cat. no. CSB-E04724 h) and TGF- $\beta$ (cat. no. CSB-E04725 h) were detected using ELISA kits, while the hydrogen peroxide $\left(\mathrm{H}_{2} \mathrm{O}_{2}\right)$ and lactate dehydrogenase (LDH) levels were examined using chemical colorimetry. ELISA and colorimetry kits were purchased from Cusabio Biotech Co., Ltd. and Nanjing Jiancheng Bioengineering Institute, respectively, and experiments were conducted according to the manufacturer's protocol.

Western blot analysis. Western blot analysis was performed to detect the protein expression levels of PRR and HO-1 in HUVEs. Briefly, the cells were lysed with RIPA buffer (Abcam), and the supernatant was collected by centrifugation at $12,000 \mathrm{x}$ g for $15 \mathrm{~min}$ at $4^{\circ} \mathrm{C}$. Protein concentration was subsequently determined by BCA assay, and then electrophoresis was conducted with a 10\% SDS-PAGE gel. Next, the samples were transferred to a PVDF membrane (Bio-Rad Laboratories, Inc.) by a Trans-Blot Transfer system (Bio-Rad Laboratories, Inc.), and the membrane was blocked with 5\% non-fat milk for $2 \mathrm{~h}$ at room temperature. The anti-PRR (cat. no. ab106834; Abcam; dilution, 1:800) and anti-HO-1 (cat. no. ab13248; Abcam; dilution, 1:700) primary antibodies were added following the manufacturer's protocol, and the samples were shaken at room temperature for $2 \mathrm{~h}$, followed by incubation at $4^{\circ} \mathrm{C}$ for $12 \mathrm{~h}$. Subsequently, the secondary antibodies rabbit anti-human IgG (cat. no. ab6759; Abcam; dilution, 1:8,000) and goat anti-rabbit IgG (cat. no. ab6721; Abcam; dilution, 1:10,000) were added, and incubated at room temperature for $1.5 \mathrm{~h}$. Chemiluminescence detection was finally conducted using an ECL reagent (Huiying, Shanghai, China). The results were quantified using the Bio-Rad ChemiDoc system
Table I. Primer sequences.

\begin{tabular}{llc}
\hline Gene & \multicolumn{1}{c}{ Sequence (5'-3') } & $\begin{array}{c}\text { Product } \\
\text { size (bp) }\end{array}$ \\
\hline PRR & $\begin{array}{l}\text { Forward: ATTGGCCTATACCAGGAGAG } \\
\text { Reverse: TTCCCCACAACGCTTCCCAA }\end{array}$ & 209 \\
HO-1 & $\begin{array}{l}\text { Forward: TTGCCAGTGCCACCAAGTTC } \\
\text { Reverse: TCAGCAGCTCCTGCAACTCC }\end{array}$ & \\
& 242 \\
$\beta$-actin & Forward: GCTGCGTGTGGCCCCTGAG & 252 \\
& Reverse: ACGCAGGATGGCATGAGGGA & \\
\hline
\end{tabular}

PRR, renin/prorenin receptor; HO-1, heme oxygenase 1.

(Bio-Rad Laboratories, Inc.) with Image Lab software version 6.0 (Bio-Rad Laboratories, Inc.).

$R T-q P C R$ analysis. RT-qPCR was used to detect the PRR and HO-1 mRNA expression levels in HUVECs. The cells were triturated and lysed using TRIzol reagent (Thermo Fisher Scientific, Inc.), and the total RNA was then collected using an RNA extraction kit (Promega Corporation). RNA concentration was measured using a UV spectrophotometer (NanoDrop One Microvolume UV-Vis spectrophotometer; Thermo Fisher Scientific, Inc.). Next, an RT kit (Takara Bio, Inc., Tokyo, Japan) was used to synthesize cDNA. The RT reaction conditions were set at $37^{\circ} \mathrm{C}$ for $15 \mathrm{~min}$, and reverse transcriptase inactivation was conducted at $85^{\circ} \mathrm{C}$ for $15 \mathrm{sec}$. Subsequently, qPCR experiments were performed with the SYBR Premix Ex Taq ${ }^{\mathrm{TM}}$ Real-Time PCR kit (Takara Bio, Inc.) according to the manufacturer's protocol. qPCR was performed by initially activating the DNA polymerase at $95^{\circ} \mathrm{C}$ for $5 \mathrm{~min}$, followed by 40 cycles of two-step PCR $\left(95^{\circ} \mathrm{C}\right.$ for $10 \mathrm{sec}$ and $60^{\circ} \mathrm{C}$ for $30 \mathrm{sec}$ ) and a final extension at $75^{\circ} \mathrm{C}$ for $10 \mathrm{~min}$, and then held at $4^{\circ} \mathrm{C}$. All primers used in qPCR were obtained from Genewiz (Suzhou, China) and are listed in Table I. The formula $2^{-\Delta \Delta C q}(21)$ was implemented to analyze the mRNA expression levels.

Statistical analysis. Data are presented as the mean \pm standard deviation. Differences between the different groups were assessed by one-way analysis of variance, followed by Dunnett's post-hoc test. Differences were analyzed by GraphPad Prism version 6 software (GraphPad Software, Inc., La Jolla, CA, USA), with $\mathrm{P}<0.05$ considered to denote differences that were statistically significant.

\section{Results}

Comparison of basic data between the two patient groups. As shown in Table II, the body mass index (BMI) and Rep in the observation patient group were significantly increased compared with those in the control group, while the HO-1 expression of the observation group was markedly reduced $(\mathrm{P}<0.05)$. However, other indices did not reveal statistical significance. Furthermore, logistic regression analysis demonstrated that HO-1 and Rep levels were independent risk factors for patients with hypertension complicated with depression 
Table II. Comparison of basic data between the two groups.

\begin{tabular}{|c|c|c|c|c|}
\hline Parameter & Hypertension with depression & Hypertension & $\mathrm{t} / \chi^{2}$ value & P-value \\
\hline Age (years) & $51.78 \pm 3.26$ & $51.36 \pm 3.92$ & 0.758 & 0.450 \\
\hline \multicolumn{5}{|l|}{ Sex } \\
\hline Male & $35(46.67 \%)$ & $40(53.33 \%)$ & $0.596^{\mathrm{a}}$ & 0.440 \\
\hline Female & $50(52.63 \%)$ & $45(47.37 \%)$ & & \\
\hline BMI $\left(\mathrm{kg} / \mathrm{m}^{2}\right)$ & $25.77 \pm 2.29$ & $25.07 \pm 1.91$ & 2.164 & 0.032 \\
\hline Disease duration (years) & $8.85 \pm 5$ & $9.52 \pm 5.19$ & 0.857 & 0.393 \\
\hline SyP (mmHg) & $156.93 \pm 9.59$ & $154.13 \pm 9.51$ & 1.911 & 0.058 \\
\hline $\mathrm{DiP}(\mathrm{mmHg})$ & $96.5 \pm 10.88$ & $95.85 \pm 7.66$ & 0.450 & 0.653 \\
\hline $\mathrm{HO}-1(\mu \mathrm{g} / \mathrm{l})$ & $1.08 \pm 0.12$ & $1.15 \pm 0.16$ & 3.227 & 0.002 \\
\hline $\operatorname{ReP}(\mu \mathrm{g} / \mathrm{l})$ & $2.46 \pm 1.30$ & $1.61 \pm 1.11$ & 4.621 & $<0.001$ \\
\hline Cor (nmol/l) & $357.48 \pm 155.27$ & $316.52 \pm 154.47$ & 1.724 & 0.087 \\
\hline $\mathrm{TG}(\mathrm{mmol} / \mathrm{l})$ & $1.35 \pm 0.28$ & $1.27 \pm 0.27$ & 1.896 & 0.060 \\
\hline $\mathrm{TC}(\mathrm{mmol} / \mathrm{l})$ & $4.58 \pm 0.63$ & $4.6 \pm 0.65$ & 0.204 & 0.839 \\
\hline LDL-C (mmol/l) & $2.56 \pm 0.44$ & $2.52 \pm 0.38$ & 0.634 & 0.527 \\
\hline HDL-C (mmol/l) & $1.27 \pm 0.20$ & $1.28 \pm 0.23$ & 0.303 & 0.763 \\
\hline Cre $(\mu \mathrm{mol} / \mathrm{l})$ & $76.59 \pm 16.38$ & $73.56 \pm 17.76$ & 1.156 & 0.249 \\
\hline $\operatorname{ALT}(\mathrm{U} / \mathrm{l})$ & $25.15 \pm 12.13$ & $24.74 \pm 10.70$ & 0.234 & 0.816 \\
\hline AST (U/l) & $25.27 \pm 6.96$ & $25.35 \pm 5.79$ & 0.082 & 0.935 \\
\hline
\end{tabular}

Data are expressed as the mean \pm standard deviation. adata are calculated using $\chi^{2}$. BMI, body mass index; HO-1, heme oxygenase 1; SyP, systolic pressure; DiP, diastolic pressure; ReP, renin precursor; Cor, cortisol; TG, triglyceride; TC, total cholesterol; LDL-C, low density lipoprotein cholesterol; HDL-C, high density lipoprotein cholesterol; Cre, creatinine; ALT, alanine aminotransfease; AST, glutamic oxalacetic transaminase.

$(\mathrm{P}<0.05$; Table III). These findings suggested that low HO-1 and high Rep levels are associated with hypertension in combination with depression. PRR was not monitored as Rep is the non-active proenzyme form of renin, the concentration of which is 5-10x that of renin in blood (22).

Cell verification. As shown in Fig. 1A, it was observed that the cells used in the experiments of the current study were polygonal. Following factor VIII immunofluorescence staining, green fluorescence was densely distributed around the nucleus and factor VIII-associated antigen was positive (Fig. 1B). These observations verified that the cells were HUVECs.

Effects of ox-LDL on cell proliferation and siPRR transfection efficiency. To investigate the effects of ox-LDL on HUVECs, the CCK-8 method was used to detect their proliferation following treatment with various ox-LDL concentrations for various time periods. The results demonstrated that ox-LDL reduced the proliferation of HUVECs in a dose-dependent manner and that cell viability increased with the prolongation of incubation time (Fig. 2A). The results also revealed that the levels of PRR protein and mRNA were significantly downregulated in the cells following siPRR plasmid transfection (Fig. 2B-D).

Effects of ox-LDL and PRR gene silencing on cells. To further explore the effects of ox-LDL on cell proliferation and its mechanism, five experimental groups were set up as follows: Control, ox-LDL, ox-LDL + mock, ox-LDL + siPRR
Table III. Logistic regression analysis.

\begin{tabular}{lrcc}
\hline Factors & P-value & Exp (B) & $95 \%$ CI \\
\hline BMI & 0.122 & 1.136 & $0.967-1.335$ \\
SyP & 0.136 & 1.028 & $0.991-1.065$ \\
HO-1 & 0.036 & 0.061 & $0.005-0.830$ \\
ReP & $<0.001$ & 1.808 & $1.350-2.423$ \\
Cor & 0.211 & 1.001 & $0.999-1.004$ \\
TG & 0.060 & 3.516 & $0.947-13.059$ \\
\hline
\end{tabular}

CI, confidence interval; BMI, body mass index; SyP, systolic pressure; HO-1, heme oxygenase 1; ReP, renin precursor; Cor, cortisol; TG, triglyceride.

and siPRR groups. The results indicated that the OD value in the ox-LDL and ox-LDL + mock groups at 48 and $72 \mathrm{~h}$ was significantly decreased compared with that in the control group, while the OD value in the ox-LDL + siPRR and siPRR groups was higher than that in the ox-LDL + mock group (Fig. 3A). This suggested that downregulation of PRR gene expression was able to promote HUVEC growth. Furthermore, the results showed that the levels of PRR protein and mRNA in the ox-LDL and ox-LDL + mock groups were significantly elevated when compared with those in the control group, while the levels of HO-1 protein and mRNA were markedly reduced in these two groups. By contrast, the levels of PRR protein and 
A
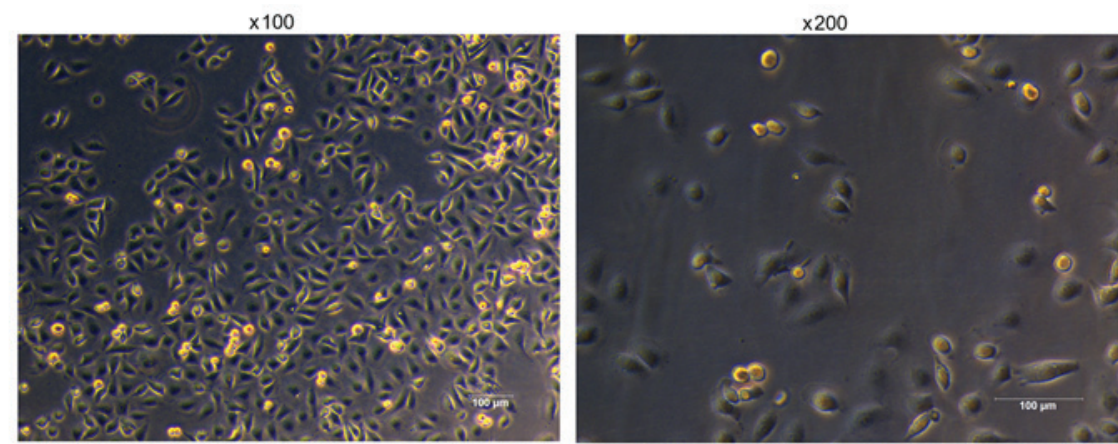

B

VIII
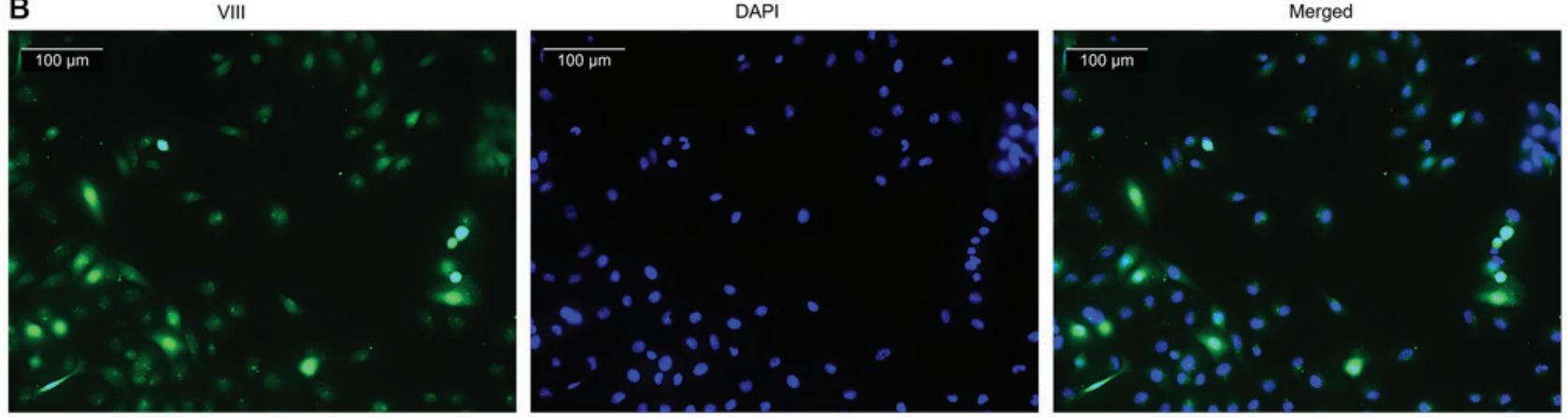

Figure 1. Observation of human umbilical vein endothelial cells. (A) Cell morphology was observed under a microscope at magnification of x100 and x200. (B) Expression of factor VIII in the cells was detected using the immunofluorescence technique.
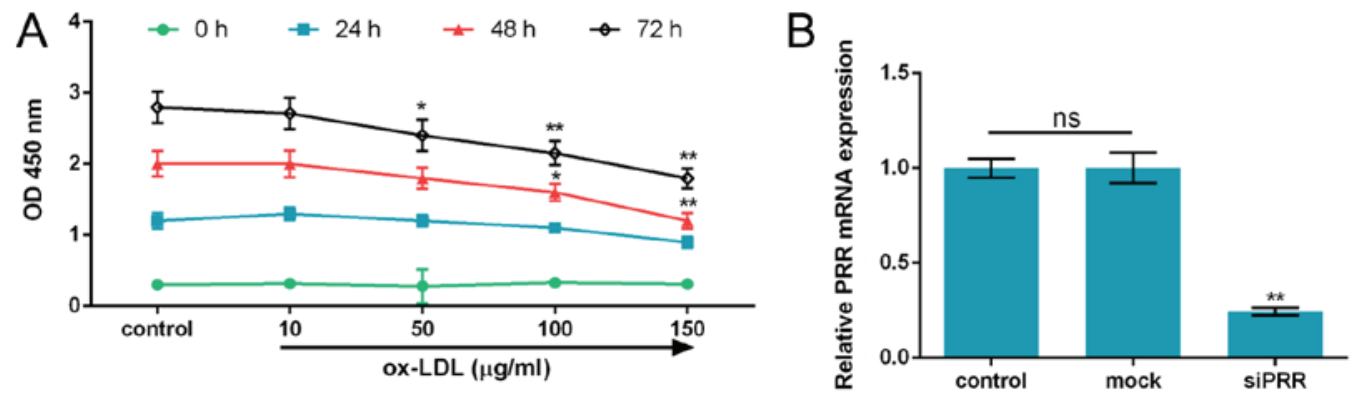

C
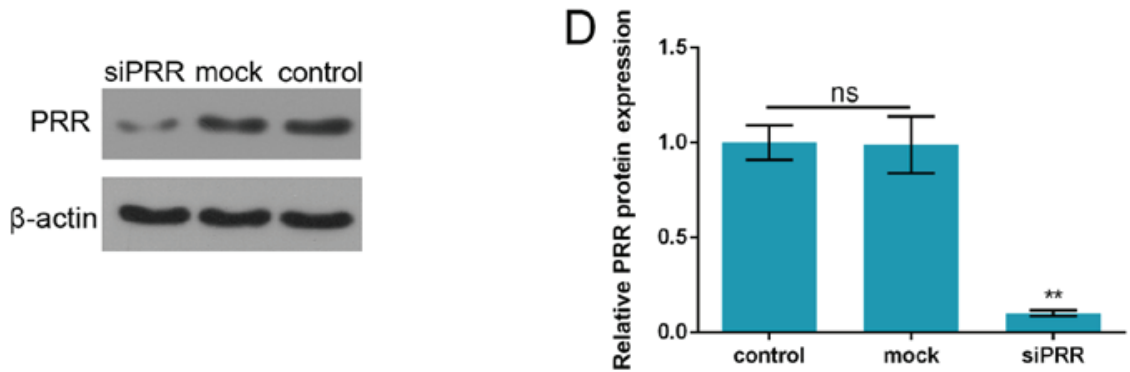

Figure 2. Effects of ox-LDL on cell proliferation and siPRR transfection efficiency. (A) Cell Counting Kit-8 assay was used to detect the proliferation of human umbilical vein endothelial cells at different concentrations of ox-LDL and different incubation times. (B) PRR mRNA expression following siPRR transfection was examined by reverse transcription-quantitative polymerase chain reaction. (C) Western blot images and (D) quantified protein expression of PRR following siPRR transfection. " $\mathrm{P}<0.05$ and ${ }^{* *} \mathrm{P}<0.01$, vs. control group. ox-LDL, oxidized low-density lipoprotein; PRR, renin/prorenin receptor; siPRR, small interfering RNA-PRR; OD, optical density; ns, not significant.

mRNA were significantly lower in the ox-LDL + siPRR and siPRR groups as compared with those in the ox-LDL + mock group, whereas that the levels of HO-1 protein and mRNA were significantly higher (Fig. 3B-F). These observations suggested that ox-LDL was able to upregulate the PRR gene expression and downregulate the HO-1 gene expression.
Effects of ox-LDL and PRR gene silencing on oxidative stress levels. The results revealed that the levels of $\mathrm{LDH}, \mathrm{H}_{2} \mathrm{O}_{2}$ and ROS in the ox-LDL and ox-LDL + mock groups were significantly higher than those in the control group. However, LDH, $\mathrm{H}_{2} \mathrm{O}_{2}$ and ROS levels in the ox-LDL + siPRR and siPRR groups were significantly reduced compared with those in the 
A

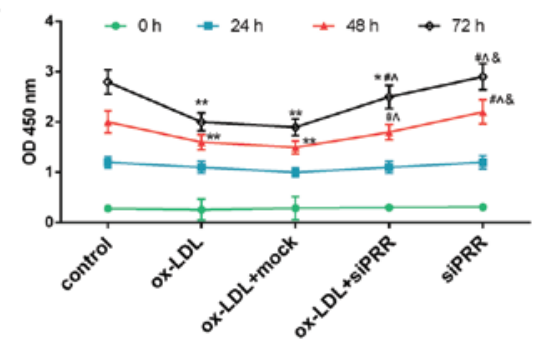

D

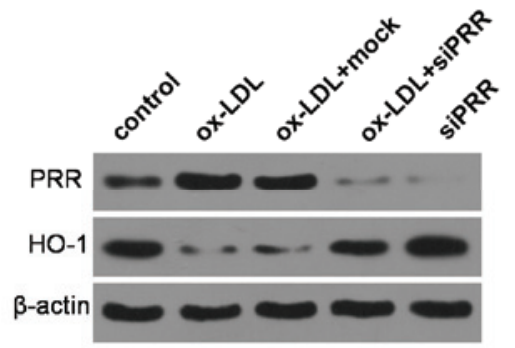

B

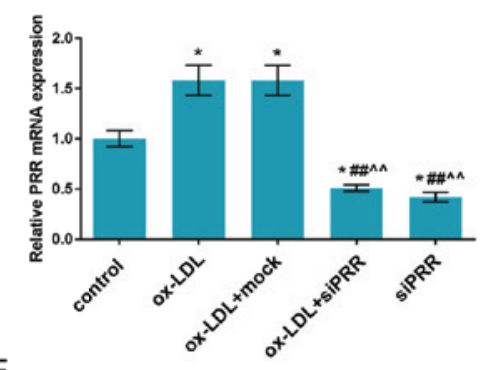

E

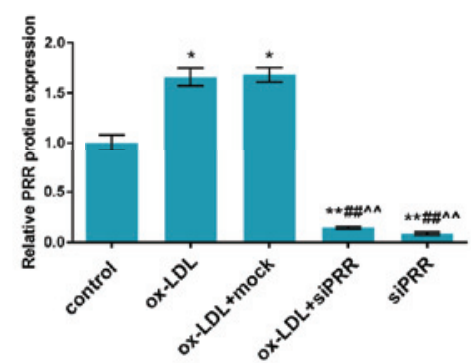

C

$\mathrm{F}$
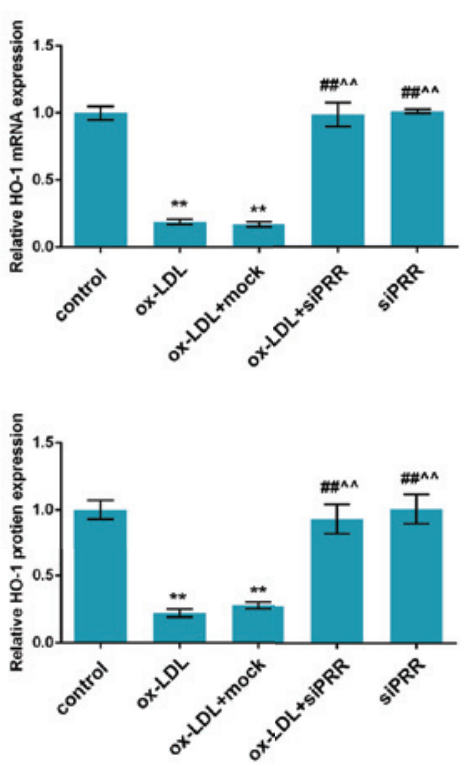

Figure 3. Effects of ox-LDL and PRR gene silencing on human umbilical vein endothelial cells. (A) Cell Counting Kit- 8 assay was performed to test the cell proliferation in the five groups. (B) PRR and (C) HO-1 mRNA expression levels in the five groups were tested by reverse transcription-quantitative polymerase chain reaction. (D) Western blot images, and quantified (E) PRR and (F) HO-1 protein expression levels. ${ }^{*} \mathrm{P}<0.05$ and ${ }^{* *} \mathrm{P}<0.01$, vs. control group; ${ }^{\#} \mathrm{P}<0.05$ and

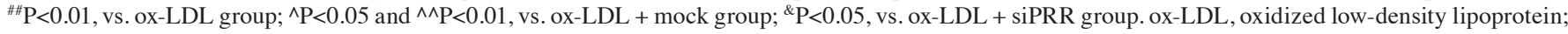
PRR, renin/prorenin receptor; HO-1, heme oxygenase 1; siPRR, small interfering RNA-PRR; OD, optical density; ns, not significant.

ox-LDL + mock group. Furthermore, the levels in the siPRR group were significantly reduced compared with those in the ox-LDL + siPRR group (Fig. 4). These findings suggested that ox-LDL increased the level of oxidative stress in HUVECs, while PRR gene silencing suppressed the promoting effect of ox-LDL and downregulated oxidative stress.

Effects of ox-LDL and PRR gene silencing on inflammatory factors. The results demonstrated that the levels of interleukin- 6 (IL-6), transforming growth factor- $\alpha$ (TGF- $\alpha$ ) and TGF- $\beta$ in the ox-LDL and ox-LDL + mock groups were increased compared with those in the control group, whereas interleukin-10 (IL-10) was downregulated in the ox-LDL and ox-LDL + mock groups. By contrast, IL-6, TGF- $\alpha$ and TGF- $\beta$ expression levels in the ox-LDL + siPRR and siPRR groups were significantly reduced compared with those in the ox-LDL + mock group, while IL-10 was upregulated. Furthermore, the levels of IL-6, TGF- $\alpha$ and TGF- $\beta$ in the siPRR group were significantly lower than those in the ox-LDL + siPRR group, while IL-10 level was significantly higher (Fig. 5). Taken together, these results suggested that ox-LDL exerted a pro-inflammatory effect in HUVECs, while PRR gene silencing inhibited the promoting effect of ox-LDL and downregulated the inflammatory response.

\section{Discussion}

Depression is a common affective disorder that causes a great disease burden to society (23). Epidemiological and biological evidence has confirmed the interaction between depression and hypertension, while genetic and epigenetic studies have also reported that there may be certain related mechanisms of action (24-26). It has been demonstrated that a significant proportion of patients with hypertension also suffer from depression, and that the blood pressure of depressed hypertension patients is higher than that of patients suffering from hypertension alone (27). To further explore the possible association between hypertension and depression, the present study compared the basic data of 170 hypertension patients with or without depression. The results revealed that there were significant differences in terms of BMI, Rep and HO-1 levels between the two groups. Further logistic regression analysis also suggested that HO-1 and Rep levels were independent factors affecting hypertension patients with depression. A study by Robaczewska et al (28) also reported that serum HO-1 was significantly elevated in patients with hypertension and depression in comparison with that in patients with depression alone.

HO-1 is the starting enzyme in the process of heme degradation and serves an important role in regulating endothelial cell integrity and oxidative stress (29). A previous study has reported that low HO-1 level increased the body's sensitivity to oxidative damage (30). RAS is an important mechanism in the development and progression of atherosclerosis and hypertension. After the combination of PRR and ligand, a large amount of Ang II can cause vasoconstriction, while RAS can also lead to an inflammatory reaction $(31,32)$. ox-LDL, an LDL peroxidation product with strong cytotoxicity, causes an increase in oxidative stress, while it can also induce the maturation of macrophages, thereby releasing inflammatory factors, such as IL-6 and TGF, and exerting pro-inflammatory effects $(33,34)$.

In a previous study, it was reported that ox-LDL was able to upregulate the expression of PRR and promote RAS (35). Similarly, the present study also demonstrated that ox-LDL reduced the proliferation of HUVECs, and upregulated the expression levels of PRR mRNA and protein in a dose-dependent manner. This was consistent with the findings of previous studies, and suggested that RAS may be activated by upregulating PRR using ox-LDL. Prorenin binds to PRR and causes 
A
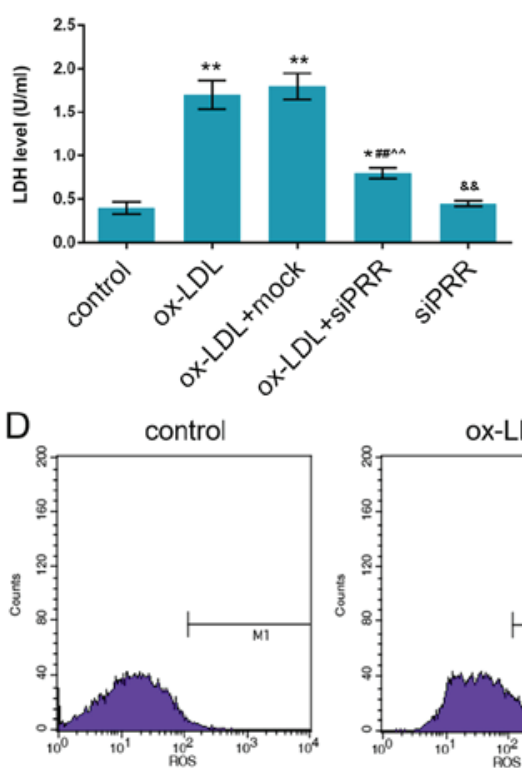

B

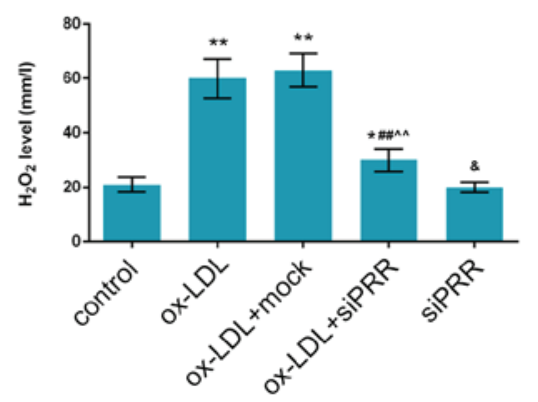

C

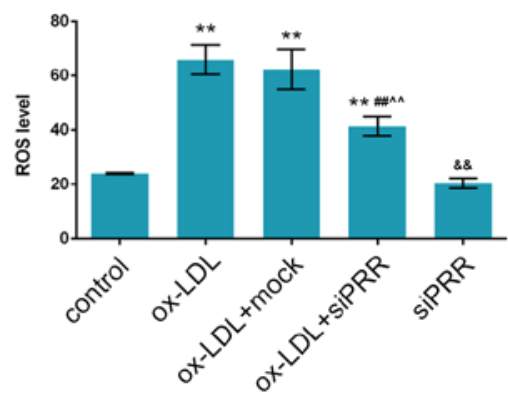

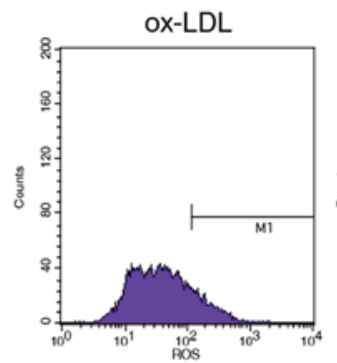
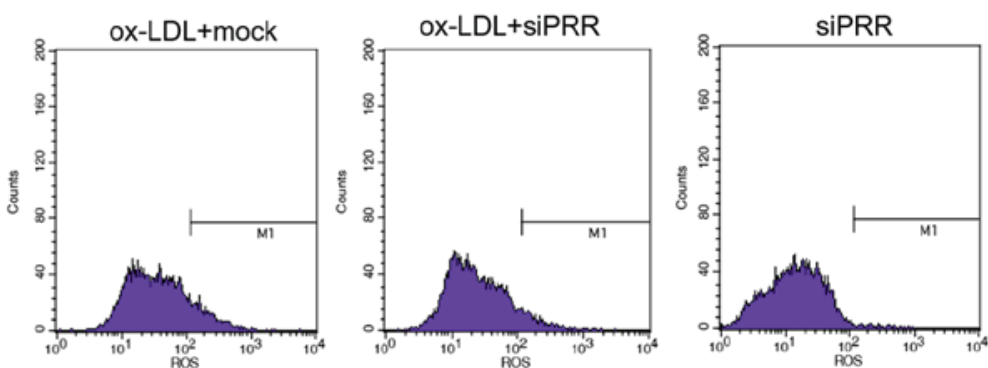

Figure 4. Effects of ox-LDL and PRR gene silencing on oxidative stress levels. (A) LDH and (B) $\mathrm{H}_{2} \mathrm{O}_{2}$ concentrations were detected using chemical colorimetry. (C) ROS levels were tested by flow cytometry. (D) Flow cytometry results for the different groups are shown. ${ }^{*} \mathrm{P}<0.05$ and ${ }^{* *} \mathrm{P}<0.01$, vs. control group; ${ }^{\# \#} \mathrm{P}<0.01$, vs. ox-LDL group; ${ }^{\wedge} \mathrm{P}<0.01$, vs. ox-LDL + mock groups; ${ }^{\circledR} \mathrm{P}<0.05$ and ${ }^{\&} \mathrm{P}<0.01$, vs. ox-LDL + siPRR group. ox-LDL, oxidized low-density lipoprotein; $\mathrm{PRR}$, renin/prorenin receptor; siPRR, small interfering RNA-PRR; LDH, lactate dehydrogenase; $\mathrm{H}_{2} \mathrm{O}_{2}$, hydrogen peroxide; ROS, reactive oxygen species.

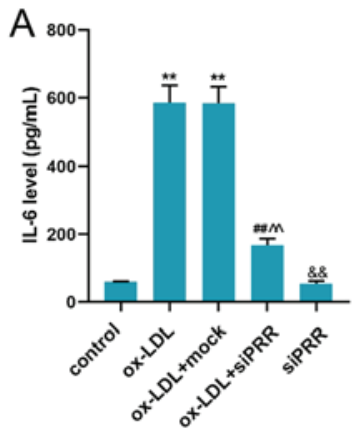

B

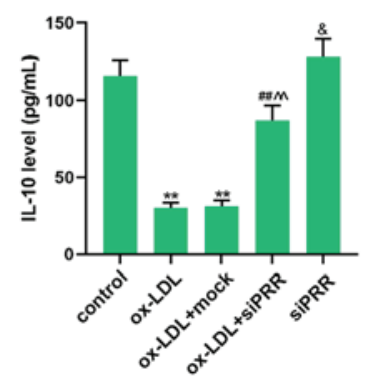

C

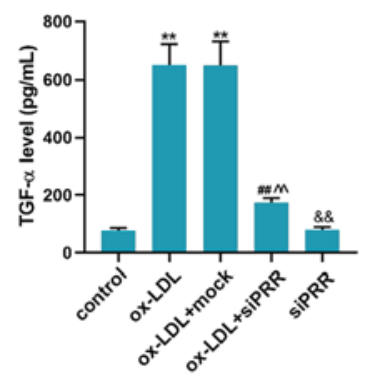

D

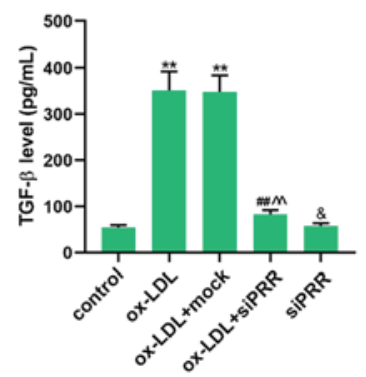

Figure 5. Effects of ox-LDL and PRR gene silencing on inflammatory factors. (A) IL-6 and (B) IL-10, and (C) TGF- $\alpha$ and (D) TGF- $\beta$ levels were detected by ELISA. ${ }^{* *} \mathrm{P}<0.01$, vs. control group; ${ }^{\# \prime} \mathrm{P}<0.01$, vs. ox-LDL group; ${ }^{\wedge} \mathrm{P}<0.01$, vs. ox-LDL + mock groups; ${ }^{\&} \mathrm{P}<0.05$ and ${ }^{\text {\&\&}} \mathrm{P}<0.01$, vs. ox-LDL + siPRR group. ox-LDL, oxidized low-density lipoprotein; PRR, renin/prorenin receptor; siPRR, small interfering RNA-PRR; IL, interleukin; TGF, transforming growth factor.

the conformational change of prorenin. This results in the activation of prorenin, leading to the increased production of Ang I. The combination of renin and PRR can significantly enhance the activity of renin and increase the production of Ang II and aldosterone, thereby activating the RAS system and enhancing its activity, ultimately leading to hypertension and inflammation (36).

The current study results further revealed that ox-LDL reduced the level of HO-1, while PRR gene silencing increased the expression of HO-1. Oxidative stress is caused by an imbalance between intracellular oxidants and antioxidants. LDH, $\mathrm{H}_{2} \mathrm{O}_{2}$ and ROS are commonly used as indicators to detect oxidative stress injury in cells, and elevated levels of these indicators represent the severity of oxidative stress injury in the cells (37). Further studies have reported that ox-LDL increased the level of oxidative stress and promoted the expression of pro-inflammatory factors, such as IL-6 and TGF.
Inhibition of PRR gene expression in the present study led to downregulation of $\mathrm{LDH}, \mathrm{H}_{2} \mathrm{O}_{2}$ and ROS levels, and inhibition of the secretion of pro-inflammatory factors. This may be associated with the upregulation of HO-1 expression by PRR gene silencing. Furthermore, conjugated diene is one of the markers commonly used to detect oxidative stress; however, the formation of this marker was not detected herein, which poses a limitation to the present study.

HO-1 is an important player against oxidative stress in mammals and has a protective effect on blood vessels in hypertension patients. Studies have demonstrated that HO-1 may be involved in the regulation of vascular endothelial function and inflammatory response by interacting with Ang II $(38,39)$. An earlier study investigating HO-1 in depression found that HO-1 had a neuroprotective effect in neurodegenerative diseases, such as Alzheimer's disease (40). Another recent study reported that the neuroprotective effect of the tricyclic antidepressant 
desipramine was associated with increased HO-1 expression (41). Furthermore, HO is considered to have the effect of reducing neuroinflammatory response $(42,43)$. Taken together, these findings suggest that the decrease in HO-1 levels may be involved in the development and progression of hypertension patients with depression, and that the PRR gene has a regulatory effect on the expression level of HO-1. However, there are certain limitations in the present study, since only in vitro model experiments were performed to examine the effect of HO-1 and PRR on inflammatory response and oxidative stress. The study findings need to be further explored and confirmed by conducting in vivo experiments.

In conclusion, the present study reported that serum HO-1 levels were significantly downregulated in hypertension patients with depression. ox-LDL served an important role in regulating the expression levels of PRR and $\mathrm{HO}-1$, and induced oxidative stress and inflammatory response by upregulating PRR expression and downregulating HO-1 expression. In addition, PRR gene silencing inhibited the ox-LDL-induced inflammatory response and oxidative stress in HUVECs. Thus, the PRR gene may be involved in the development of hypertension and depression by downregulating the expression of HO-1, and increasing the oxidative stress and inflammatory responses. Regulating the expression levels of HO-1 and PRR to inhibit the oxidative stress and pro-inflammatory effect of ox-LDL may provide a novel approach for the treatment of hypertension patients with depression.

\section{Acknowledgements}

Not applicable.

\section{Funding}

No funding was received.

\section{Availability of data and materials}

The analyzed data sets generated during the present study are available from the corresponding author on reasonable request.

\section{Authors' contributions}

Substantial contributions to conception and design were made by XG, CL and HW. Data acquisition, analysis and interpretation were conducted by JF, CJ and YZ. The article was drafted or critically revised for important intellectual content by $\mathrm{YZ}$ and HW. YZ and JF agreed to be accountable for all aspects of the work in ensuring that questions related to the accuracy or integrity of the work are appropriately investigated and resolved. All authors provided final approval of the manuscript version to be published.

\section{Ethics approval and consent to participate}

All procedures performed in studies involving human participants were in accordance with the ethical standards of the committee of the Affiliated Yantai Yuhuangding Hospital of Qingdao University and with the 1964 Helsinki Declaration and its later amendments, or comparable ethical standards.

\section{Patient consent for publication}

Not applicable.

\section{Competing interests}

The authors declare that they have no competing interests.

\section{References}

1. Kretchy IA, Owusu-Daaku FT and Danquah SA: Mental health in hypertension: Assessing symptoms of anxiety, depression and stress on anti-hypertensive medication adherence. Int J Ment Health Syst 8: 25, 2014.

2. Cene CW, Halladay JR, Gizlice Z, Roedersheimer K, Hinderliter A, Cummings DM, Donahue KE, Perrin AJ and DeWalt DA: Associations between subjective social status and physical and mental health functioning among patients with hypertension. J Health Psychol 21: 2624-2635, 2016.

3. Li Z, Li Y, Chen L, Chen P and Hu Y: Prevalence of depression in patients with hypertension: A systematic review and meta-analysis. Medicine (Baltimore) 94: e1317, 2015.

4. Almas A, Patel J, Ghori U, Ali A, Edhi AI and Khan MA: Depression is linked to uncontrolled hypertension: A case-control study from Karachi, Pakistan. J Ment Health 23: 292-296, 2014.

5. Chen Y, Guo F, Ru Z, Kong H, Sun H, Yu H, Yang W, Zhang Q and Zhou M: Nuclear factor- $\kappa \mathrm{B}$ signaling negatively regulates high glucose-induced vascular endothelial cell damage downstream of the extracellular signal-regulated kinase/c-Jun N-terminal kinase pathway. Exp Ther Med 14: 3851-3855, 2017.

6. Ando J and Kamiya A: Blood flow and vascular endothelial cell function. Front Med Biol Eng 5: 245-264, 1993.

7. Schulz E, Gori T and Münzel T: Oxidative stress and endothelial dysfunction in hypertension. Hypertens Res 34: 665-673, 2011.

8. Dinh QN, Drummond GR, Sobey CG and Chrissobolis S: Roles of inflammation, oxidative stress, and vascular dysfunction in hypertension. Biomed Res Int 2014: 406960, 2014.

9. Crowley SD: The cooperative roles of inflammation and oxidative stress in the pathogenesis of hypertension. Antioxid Redox Signal 20: 102-120, 2014.

10. Wolkowitz OM, Mellon SH,EpelES, Lin J,DhabharFS, Su Y, Reus VI, Rosser R, Burke HM, Kupferman E, et al: Leukocyte telomere length in major depression: Correlations with chronicity, inflammation and oxidative stress-preliminary findings. PLoS One 6: e17837, 2011.

11. Czarny P, Wigner P, Galecki P and Sliwinski T: The interplay between inflammation, oxidative stress, DNA damage, DNA repair and mitochondrial dysfunction in depression. Prog Neuropsychopharmacol Biol Psychiatry 80: 309-321, 2018.

12. Carey RM: The intrarenal renin-angiotensin system in hypertension. Adv Chronic Kidney Dis 22: 204-210, 2015.

13. Gupta S, Chattopadhyaya I, Agrawal BK, Sehajpal PK and Goel RK: Correlation of renin angiotensin system (RAS) candidate gene polymorphisms with response to ramipril in patients with essential hypertension. J Postgrad Med 61: 21-26, 2015.

14. Murck H, Schussler P and Steiger A: Renin-angiotensinaldosterone system: The forgotten stress hormone system: Relationship to depression and sleep. Pharmacopsychiatry 45: 83-95, 2012.

15. Peng H, Li W, Seth DM, Nair AR, Francis J and Feng Y: (Pro)renin receptor mediates both angiotensin II-dependent and -independent oxidative stress in neuronal cells. PLoS One 8: e58339, 2013

16. Li W, Liu J, Hammond SL, Tjalkens RB, Saifudeen Z and Feng Y: Angiotensin II regulates brain (pro)renin receptor expression through activation of cAMP response element-binding protein. Am J Physiol Regul Integr Comp Physiol 309: R138-R147, 2015.

17. Shen Y, Yang T, Guo S, Li X, Chen L, Wang T and Wen F: Increased serum ox-LDL levels correlated with lung function, inflammation, and oxidative stress in COPD. Mediators Inflamm 2013: 972347, 2013

18. Le WD, Xie WJ and Appel SH: Protective role of heme oxygenase-1 in oxidative stress-induced neuronal injury. J Neurosci Res 56: 652-658, 1999.

19. Innes K, Hooper J, Bramley M and DahDah P: Creation of a clinical classification. International statistical classification of diseases and related health problems-10th revision, Australian modification (ICD-10-AM). Health Inf Manag 27: 31-38, 1997. 
20. Jaffe EA, Nachman RL, Becker CG and Minick CR: Culture of human endothelial cells derived from umbilical veins. Identification by morphologic and immunologic criteria. J Clin Invest 52: 2745-2756, 1973.

21. Livak KJ and Schmittgen TD: Analysis of relative gene expression data using real-time quantitative PCR and the 2(-Delta Delta C(T)) method. Methods 25: 402-408, 2001.

22. Nguyen G and Danser AH: Prorenin and (pro)renin receptor: A review of available data from in vitro studies and experimental models in rodents. Exp Physiol 93: 557-563, 2008.

23. Lee AL, Ogle WO and Sapolsky RM: Stress and depression: Possible links to neuron death in the hippocampus. Bipolar Disord 4: 117-128, 2002.

24. Grimsrud A, Stein DJ, Seedat S, Williams D and Myer L: The association between hypertension and depression and anxiety disorders: Results from a nationally-representative sample of South African adults. PLoS One 4: e5552, 2009.

25. Löwe B, Gräfe K, Ufer C, Kroenke K, Grünig E, Herzog W, Borst MM: Anxiety and depression in patients with pulmonary hypertension. Psychosom Med 66: 831-836, 2004.

26. Scalco AZ, Scalco MZ, Azul JB and Lotufo Neto F: Hypertension and depression. Clinics (Sao Paulo) 60: 241-250, 2005.

27. Meng L, Chen D, Yang Y, Zheng Y and Hui R: Depression increases the risk of hypertension incidence: A meta-analysis of prospective cohort studies. J Hypertens 30: 842-851, 2012.

28. Robaczewska J, Kedziora-Kornatowska K, Kucharski R, Nowak M, Muszalik M, Kornatowski $M$ and Kędziora J: Decreased expression of heme oxygenase is associated with depressive symptoms and may contribute to depressive and hypertensive comorbidity. Redox Rep 21: 209-218, 2016.

29. Zhao Y, Zhang L, Qiao Y, Zhou X, Wu G, Wang L, Peng Y, Dong X, Huang H, Si L, et al: Heme oxygenase-1 prevents cardiac dysfunction in streptozotocin-diabetic mice by reducing inflammation, oxidative stress, apoptosis and enhancing autophagy. PLoS One 8: e75927, 2013.

30. Unuma K, Aki T, Matsuda S, Funakoshi T, Yoshida K and Uemura K: Inducer of heme oxygenase-1 cobalt protoporphyrin accelerates autophagy and suppresses oxidative damages during lipopolysaccharide treatment in rat liver. Hepatol Res 43: 91-96, 2013

31. Mendonça L, Mendes-Ferreira P, Bento-Leite A, Cerqueira R, Amorim MJ, Pinho P, Brás-Silva C, Leite-Moreira AF and Castro-Chaves P: Angiotensin-(1-7) modulates angiotensin II-induced vasoconstriction in human mammary artery. Cardiovasc Drugs Ther 28: 513-522, 2014.

32. Deng Y, Deng Y, He X, Chu J, Zhou J, Zhang Q, Guo W, Huang P, Guan X, Tang Y, et al: Prenatal inflammation-induced $\mathrm{NF}-\mathrm{kB}$ dyshomeostasis contributes to renin-angiotensin system over-activity resulting in prenatally programmed hypertension in offspring. Sci Rep 6: 21692, 2016.

33. Hu LH, Yu Y, Jin SX, Nie P, Cai ZH, Cui ML, Sun SQ, Xiao H, Shao Q, Shen LH and He B: Orphan nuclear receptor Nur77 Inhibits Oxidized LDL-induced differentiation of RAW264.7 murine macrophage cell line into dendritic like cells. BMC Immunol 15: 54, 2014.
34. Liu W, Yin Y, Zhou Z, He M and Dai Y: OxLDL-induced IL-1 beta secretion promoting foam cells formation was mainly via CD36 mediated ROS production leading to NLRP3 inflammasome activation. Inflamm Res 63: 33-43, 2014.

35. Catar RA, Muller G, Heidler J, Schmitz G, Bornstein SR and Morawietz H: Low-density lipoproteins induce the renin-angiotensin system and their receptors in human endothelial cells. Horm Metab Res 39: 801-805, 2007.

36. Ferrario CM and Mullick AE: Renin angiotensin aldosterone inhibition in the treatment of cardiovascular disease. Pharmacol Res 125: 57-71, 2017.

37. Qiao H, Chen H, Dong Y, Ma H, Zhao G, Tang F and Li Z: Polydatin attenuates $\mathrm{H} 2 \mathrm{O} 2$-induced oxidative stress via PKC pathway. Oxid Med Cell Longev 2016: 5139458, 2016.

38. Mai J, Qiu Q, Lin YQ, Luo NS, Zhang HF, Wen ZZ, Wang JF and YangXin C: Angiotensin II-derived reactive oxygen species promote angiogenesis in human late endothelial progenitor cells through heme oxygenase-1 via ERK1/2 and AKT/PI3K pathways. Inflammation 37: 858-870, 2014.

39. Davis PA, Pagnin E, Dal Maso L, Caielli P, Maiolino G, Fusaro M, Paolo Rossi G and Calò LA: SIRT1, heme oxygenase-1 and NO-mediated vasodilation in a human model of endogenous angiotensin II type 1 receptor antagonism: implications for hypertension. Hypertens Res 36: 873-878, 2013.

40. Mancuso C, Santangelo R and Calabrese V: The heme oxygenase/biliverdin reductase system: A potential drug target in Alzheimers disease. J Biol Regul Homeost Agents 27(2 Suppl): 75-87, 2013

41. Lin HY, Yeh WL, Huang BR, Lin C, Lai CH, Lin H and Lu DY: Desipramine protects neuronal cell death and induces heme oxygenase-1 expression in Mes23.5 dopaminergic neurons. PLoS One 7: e50138, 2012.

42. Zhao CY, Lei H, Zhang Y, Li L, Xu SF, Cai J, Li PP, Wang L, Wang XL and Peng Y: L-3-n-Butylphthalide attenuates neuroinflammatory responses by downregulating JNK activation and upregulating Heme oxygenase-1 in lipopolysaccharide-treated mice. J Asian Nat Prod Res 18: 289-302, 2016.

43. Lee DS, Lee M, Sung SH and Jeong GS: Involvement of heme oxygenase-1 induction in the cytoprotective and neuroinflammatory activities of Siegesbeckia Pubescens isolated from 5,3'-dihydroxy-3,7,4'-trimethoxyflavone in HT22 cells and BV2 cells. Int Immunopharmacol 40: 65-72, 2016.

This work is licensed under a Creative Common Attribution-NonCommercial-NoDerivatives 4.0 International (CC BY-NC-ND 4.0) License. 